\title{
Investigation of PTEN promoter methylation in ameloblastoma
}

\author{
Puangwan Lapthanasupkul ${ }^{1}$, Boworn Klongnoi ${ }^{2}$, Apiwat Mutirangura ${ }^{3}$, Nakarin Kitkumthorn ${ }^{4}$
}

${ }^{1}$ DDS, MSc, PhD. Department of Oral and Maxillofacial Pathology, Faculty of Dentistry, Mahidol University, Bangkok, Thailand ${ }^{2}$ DDS, MD, DMD. Department of Oral and Maxillofacial Surgery, Faculty of Dentistry, Mahidol University, Bangkok, Thailand ${ }^{3} \mathrm{MD}$, PhD. Center of Excellence in Molecular Genetics of Cancer and Human Diseases, Department of Anatomy, Faculty of Medicine, Chulalongkorn University, Bangkok, Thailand

${ }^{4}$ DDS, PhD. Department of Oral Biology, Faculty of Dentistry, Mahidol University, Bangkok, Thailand

Correspondence:

Department of Oral Biology

Faculty of Dentistry, Mahidol University

6 Yothi Street, Bangkok, Thailand

Nakarinkit@gmail.com

Received: 10/10/2019

Accepted: 09/12/2019
Lapthanasupkul P, Klongnoi B, Mutirangura A, Kitkumthorn N. Investigation of PTEN promoter methylation in ameloblastoma. Med Oral Patol Oral Cir Bucal. 2020 Jul 1;25 (4):e481-7.

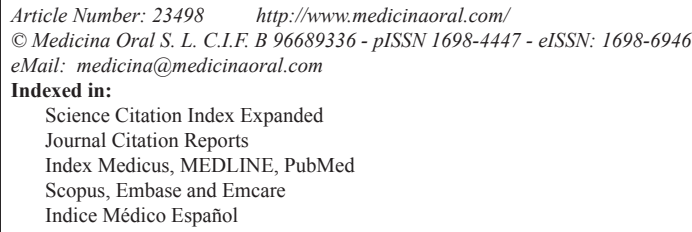

\begin{abstract}
Background: Phosphatase and tensin homolog $(P T E N)$ acts as a tumor suppressor gene. Inactivation of PTEN has been reported in various types of cancers. PTEN promoter methylation possibly underlies PTEN inactivation, which results in tumorigenesis. The aim of this study was to investigate whether PTEN promoter methylation contributes to PTEN inactivation in ameloblastoma and its associated protein expression.

Material and Methods: In total, 20 fresh-frozen ameloblastoma samples were evaluated for PTEN promoter methylation using methylation-specific polymerase chain reaction (MS-PCR). A subset of 10 paraffin-embedded ameloblastoma samples was examined for PTEN expression through immunohistochemistry. Four primary cultured ameloblastoma cells were investigated for PTEN promoter methylation and PTEN transcriptional expression via reverse transcription PCR.

Results: PTEN promoter methylation was detected in $65 \%(13 / 20)$ of the ameloblastoma samples. Of 10 ameloblastoma samples, 4 exhibited reduced PTEN expression. Of 5 samples with methylated PTEN, $3(60 \%)$ were associated with loss of PTEN expression. However, PTEN expression was detected in $4(80 \%)$ of 5 samples with unmethylated PTEN. In addition, 3 (75\%) of 4 primary ameloblastoma cell cultures exhibited an inverse correlation between PTEN promoter methylation and PTEN transcription level.

Conclusions: PTEN promoter methylation is found in a number of ameloblastomas but not significantly correlated with loss of PTEN expression. Genetic or epigenetic mechanisms other than PTEN promoter methylation may contribute to PTEN inactivation in ameloblastoma tumor cells.
\end{abstract}

Key words: PTEN, promoter methylation, ameloblastoma. 


\section{Introduction}

Ameloblastoma is the most frequently encountered neoplasm arising from the epithelium of the tooth-forming apparatus. Although this tumor is benign, it exhibits locally invasive behavior and has a high risk of recurrence. Its macroscopic features range from completely solid to multicystic appearance. Its histopathological subtypes include follicular, plexiform, acanthomatous, granular cell, basal cell, and desmoplastic ameloblastomas. In rare cases, ameloblastoma may metastasize despite its benign histology; this type of ameloblastoma is termed as metastasizing ameloblastoma (1). Ameloblastic carcinoma, a malignant counterpart of ameloblastoma, is markedly rare, with only 100 cases reported to date; this tumor exhibits cytological features of malignancy and may or may not metastasize (2).

Phosphatase and tensin homolog (PTEN) is located on chromosome 10q23.3 and has been implicated in many familial and sporadic cancers $(3,4)$. Deletions or somatic mutations in PTEN have been detected in many types of cancers, including prostate, breast, and brain cancer (3). Apart from genetic mutation, the epigenetic regulation of PTEN via differential methylation may contribute to its inactivation (5). Methylation of the PTEN promoter region has been reported in some types of cancers and has been suggested to be involved in tumorigenesis (68). In ameloblastic tumors, PTEN displayed high frequent allelic loss (62\%) (9). In addition, PTEN has been reported to be completely absent in $33.3 \%$ of ameloblastoma cases (10). We hypothesized that PTEN promoter methylation results in decreased PTEN expression in this odontogenic tumor. The aim of this study was to examine PTEN expression and investigate whether PTEN promoter methylation contributes to PTEN inactivation in ameloblastoma.

\section{Material and Methods}

\section{- Sample recruitment}

Fresh-frozen samples were obtained from 20 patients with ameloblastoma from the Department of Oral and Maxillofacial Surgery, Faculty of Dentistry, Mahidol University between January 2018 and January 2019. Some parts of the specimens were fixed in $10 \%$ buffered formalin for hematoxylin and eosin staining. Histopathological diagnosis of solid/multicystic ameloblastoma was performed by two oral pathologists (PL and NK). Furthermore, a cohort study was performed wherein 4 fresh solid/multicystic ameloblastoma tissue samples were harvested to form a primary cell culture. Table 1 displays the detailed demographic data.

Table 1: Detailed data of phosphatase and tensin homolog $(P T E N)$ promoter methylation, PTEN expression in ameloblastoma and demographic data.

\begin{tabular}{|c|c|c|c|c|c|c|}
\hline \multicolumn{7}{|c|}{ Ameloblastoma tissue } \\
\hline No & Gender & \begin{tabular}{|c|}
$\begin{array}{c}\text { Age at diagnosis } \\
\text { (yrs.) }\end{array}$ \\
\end{tabular} & Location & $\begin{array}{l}\text { Histological } \\
\text { appearance }\end{array}$ & $\begin{array}{c}P T E N \text { promoter } \\
\text { methylation }\end{array}$ & $\begin{array}{c}\text { PTEN expression } \\
\text { (Immunohistochemistry) }\end{array}$ \\
\hline AM1 & Female & 30 & Mandible & Plexiform & Unmet & Postive \\
\hline AM2 & Male & 63 & Mandible & Plexiform & Met & Postive \\
\hline AM3 & Female & 47 & Mandible & Plexiform & Met & Negative \\
\hline AM4 & Male & 11 & Maxilla & Plexiform & Unmet & Postive \\
\hline AM5 & Female & 60 & Mandible & Follicular & Unmet & Postive \\
\hline AM6 & Male & 66 & Mandible & Plexiform & Met & Postive \\
\hline AM7 & Male & 23 & Mandible & Follicular & Met & Negative \\
\hline AM8 & Male & 51 & Mandible & Follicular & Unmet & Negative \\
\hline AM9 & Male & 51 & Mandible & Follicular & Met & Negative \\
\hline AM10 & Female & 25 & Mandible & Follicular & Unmet & Postitive \\
\hline AM11 & Male & 59 & Mandible & Plexiform & Met & Not available \\
\hline AM12 & Male & 44 & Maxilla & Plexiform & Met & Not available \\
\hline AM13 & Female & 24 & Maxilla & Plexiform & Unmet & Not available \\
\hline AM14 & Male & 54 & Mandible & Follicular & Unmet & Not available \\
\hline AM15 & Female & 54 & Mandible & Plexiform & Met & Not available \\
\hline AM16 & Male & 73 & Mandible & Plexiform & Met & Not available \\
\hline AM17 & Female & 8 & Mandible & Plexiform & Met & Not available \\
\hline AM18 & Male & 66 & Mandible & Follicular & Met & Not available \\
\hline AM19 & Male & 50 & Mandible & Follicular & Met & Not available \\
\hline AM20 & Male & 50 & Maxilla & Follicular & Met & Not available \\
\hline \multicolumn{7}{|c|}{ Ameloblastoma primary cell culture } \\
\hline No & Gender & \begin{tabular}{|c|}
$\begin{array}{c}\text { Age at diagnosis } \\
\text { (yrs.) }\end{array}$ \\
\end{tabular} & Location & $\begin{array}{l}\text { Histological } \\
\text { appearance }\end{array}$ & $\begin{array}{c}\text { PTEN promoter } \\
\text { methylation }\end{array}$ & $\begin{array}{c}P T E N \text { expression } \\
\text { (reverse-transcriptase PCR) }\end{array}$ \\
\hline $\mathrm{A} 1 \mathrm{C}$ & Female & 30 & Maxilla & Follicular & Unmet & Postitve \\
\hline $\mathrm{A} 2 \mathrm{C}$ & Male & 37 & Mandible & Follicular & Unmet & Postitve \\
\hline $\mathrm{A} 3 \mathrm{C}$ & Female & 42 & Mandible & Plexiform & Met & Negative \\
\hline $\mathrm{A} 4 \mathrm{X}$ & Male & 20 & Mandible & Plexiform & Unmet & Postitve \\
\hline
\end{tabular}


The cultures were grown in Dulbecco's modified Eagle's medium supplemented with $10 \%$ fetal bovine serum (Invitrogen, Carlsbad, CA, USA) and maintained for 3-4 passages prior to DNA extraction.

- DNA extraction, bisulfite modification, and methylation-specific polymerase chain reaction (MS-PCR)

Genomic DNA was extracted using 10\% sodium dodecyl sulfate buffer and proteinase $\mathrm{K}$ at $50^{\circ} \mathrm{C}$ overnight, followed by phenol/chloroform extraction and ethanol precipitation. Subsequently, the obtained DNA was air dried, dissolved in distilled water, and quantified using a NanoDrop spectrophotometer (ND-1000 Spectrophotometer; NanoDrop Technologies, Wilmington, DE, USA). An optical density (at 260/280 ratio) of $>1.8$ is acceptable for DNA purity and PCR. Next, the DNA samples were converted by sodium bisulfite treatment using the EZ DNA Methylation-Gold ${ }^{\mathrm{TM}}$ kit (Zymo Research, Irvine, CA, USA), as per the manufacturer's instructions.

The bisulfite-treated DNA samples were then subjected to MS-PCR using primers specific for either the methylated or unmethylated forms of PTEN: 1) PTEN methylated sequence, forward 5'-GTTTGGGGATTTTTTTTTCGC-3' and reverse 5'-AACCCTTCCTACGCCGCG-3' and 2) PTEN unmethylated sequence, forward 5'-TATTAGTTTGGGGATTTTTTTTTTGT-3' and reverse 5'-CCCAACCCTTCCTACACCACA-3' (11). Both forms of PTEN were amplified with HotStarTaq (Qiagen, Tokyo, Japan) in 40 cycles at an annealing temperature of $60^{\circ} \mathrm{C}$. Aliquots of MS-PCR products (181 bp, both PCRs) were analyzed on $2 \%$ agarose gel or $8 \%$ nondenaturing acrylamide gel and then stained with SYBR green nucleic acid gel stain (Gelstar, Lonza, Allendale, NJ, USA).

For MS-PCR analysis, PTEN unmethylated primers amplified unmethylated product while PTEN methylated primers amplified only methylated product present in the ameloblastoma tissue. From our pilot study, DNA extracted from normal fibrous connective tissue was only amplified by unmethylated primers but not amplified by methylated primers. Thus, the methylated product, if present, came from ameloblastoma tumor cells. In our pilot study, we examined DNA in different cell lines and observed that the HeLa cell line had partial PTEN promoter methylation and PTEN expression. Therefore, HeLa DNA and distilled water were included as the positive and negative controls, respectively, in all experiments.

- RNA extraction and reverse transcription PCR (RT-PCR)

PTEN expression in the ameloblastoma primary cell cultures was examined using RT-PCR. Total RNA was extracted using the TRIzol reagent (Invitrogen, Singapore), as per the manufacturer's instructions. Singlestranded complementary DNA (cDNA) was synthesized using the RevertAid First Strand cDNA Synthesis kit (Thermo Fisher Scientific, Waltham, MA, USA), as per the manufacturer's instructions.

PTEN was amplified using HotStarTaq (Qiagen, Tokyo, Japan) in 40 cycles at an annealing temperature of $55^{\circ} \mathrm{C}$ using the forward primer $5^{\prime}$-GGACGAACTGGTGTAATGATATG-3' and reverse primer 5'-TCTACTGTTTTTGTGAAGTACAGC-3' (12). To investigate the relative expression of a candidate gene, glyceraldehyde-3-phosphate dehydrogenase was used as an endogenous DNA control, with the sequences of the forward and reverse primers being 5'-CTCAGACACCATGGGGAAGGTGA-3' and 5'-ATGATCTTGAGGCTGTTGTCATA-3', respectively. Both PCR mixtures contained PCR buffer $(1 \times)$ (Qiagen, Tokyo, Japan), deoxynucleotide triphosphates $(0.2 \mathrm{mM})$, the two primers (final concentration: $0.4 \mu \mathrm{M}$ ), HotStarTaq (1 U) (Qiagen, Tokyo, Japan), and template DNA (50 ng). The PCR products (PTEN: 671 bp and GADPH: $450 \mathrm{bp}$ ) were separated via gel electrophoresis on an $8 \%$ nondenaturing acrylamide gel and stained with SYBR green nucleic acid gel stain (Gelstar, Lonza, Allendale, NJ, USA).

- Immunohistochemical staining of PTEN

In this experiment, the paraffin tissues were available in only 10 cases. Formalin-fixed paraffin embedded blocks were cut into $3-\mu \mathrm{m}$ thick sections. The histological sections of the relative samples were confirmed by a pathologist. Immunohistochemistry was performed with an antihuman monoclonal antibody against PTEN Clone 6H2.1 (dilution 1:100, DAKO, Glostrup, Denmark) in Tris-HCl buffer antibody diluent (Dako, Glostrup, Denmark) on the Ventana $^{\circledR}$ Benchmarck XT (Ventana-Roche Diagnostics, Meylan, France) automated slide strainer in combination with the Ventana UltraView DAB IHC Detection $\mathrm{Kit}^{\circledR}$. Before mounting, the sections were counterstained with Hematoxylin $\mathrm{II}^{\circledR}$ for $8 \mathrm{~min}$, bluing reagent ${ }^{\circledR}$ for $4 \mathrm{~min}$, Hematoxylin II for $4 \mathrm{~min}$, and bluing reagent for $4 \mathrm{~min}$. To support the validity of staining and identify experimental artifacts, negative (omitting the primary antibody) and positive controls (normal breast tissue) were included in each run. Nuclear and cytoplasmic immunostaining of PTEN in ameloblastoma tumor cells were graded based on the presence or absence of protein staining.

- Statistical analysis

SPSS software for Windows version 22 (SPSS Inc., Chicago, IL) was used to analyze all data. The effects of age and sex of the patients as well as the histological appearance of ameloblastoma on the PTEN methylation status and PTEN expression were investigated using the Pearson's correlation coefficient test, chi-square test, and Fisher's exact test. $P<0.05$ was considered statistically significant. 


\section{Results}

- MS-PCR and immunohistochemistry of ameloblastoma tissues

We examined PTEN promoter methylation and whether it affects PTEN expression in ameloblastomas. PTEN promoter methylation was observed in $65 \%(13 / 20)$ of ameloblastoma samples (Table 1). The exemplified gel electrophoresis is demonstrated in Fig. 1. Ten samples of these ameloblastoma cases were investigated for immunohistochemical staining of PTEN. We found loss of PTEN expression in 3 of $5(60 \%)$ ameloblastoma samples with PTEN promoter methylation while PTEN expression was present in 4 of $5(80 \%)$ ameloblastoma samples with no PTEN promoter methylation (Table 1). Representative samples showing positive and negative immunostaining of PTEN were shown in Fig. 2.

- Association among PTEN promoter methylation, PTEN expression, and clinicopathological parameters Table 1 shows the association between PTEN promoter methylation and PTEN expression in the ameloblastoma samples. No significant correlation was found between $P T E N$ promoter methylation and PTEN expression $(P=$ $0.52)$. Furthermore, no correlation between $P T E N$ promoter methylation and age $(P=0.49)$, gender $(P=0.40)$, location $(P=0.62)$ and the histological appearance of ameloblastoma $(P=0.41)$ was demonstrated. Similarly, no correlation was observed between PTEN expression and age $(P=0.25)$, gender $(P=1.00)$, location $(P=0.51)$, and the histological appearance of ameloblastoma $(P=1.00)$. - MS-PCR and RT-PCR of primary ameloblastoma cell cultures

PTEN promoter methylation and PTEN expression were examined in four primary ameloblastoma cell cultures using RT-PCR (Fig. 3). Only 1 of 4 samples exhibited $P T E N$ promoter methylation and showed no PTEN transcription. PTEN promoter methylation was inversely correlated with PTEN transcription level in the remaining ameloblastoma samples (75\%) (Table 1).

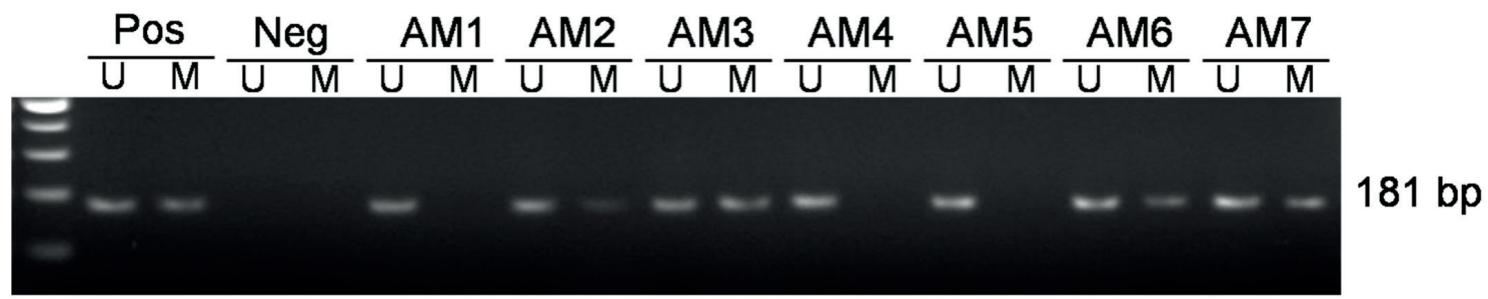

Fig. 1: MS-PCR analysis of PTEN promoter in ameloblastoma samples.

PCR products amplified using primers specific for unmethylated (U) and methylated (M) forms. AM2, AM3, AM6, and AM7 show promoter methylation. The ladder in the left lane is a 100-bp marker. Both methylated and unmethylated PCR products are 181 bp. Positive control, Pos: HeLa cell line; negative control, Neg: distilled water; unmethylated PCR products, U; methylated PCR products, M.

\section{A}

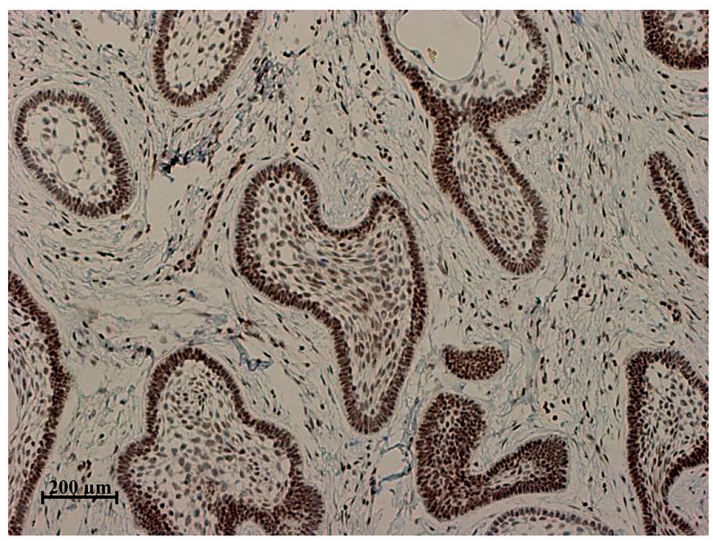

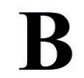

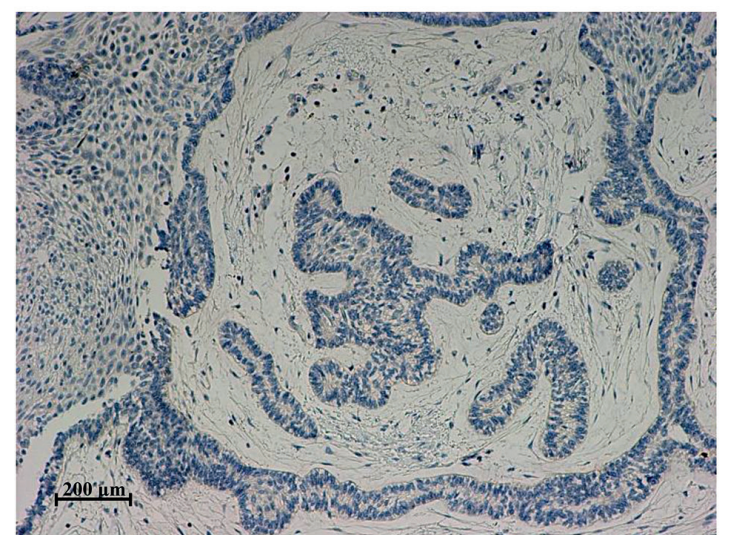

Fig. 2: (A) Immunostaining of PTEN in ameloblastoma (streptavidin-biotin; 100×). PTEN immunoexpression is observed in cytoplasm and nucleus of ameloblastoma tumor cells (B) No expression of PTEN in ameloblastoma (streptavidin-biotin, 100×). 


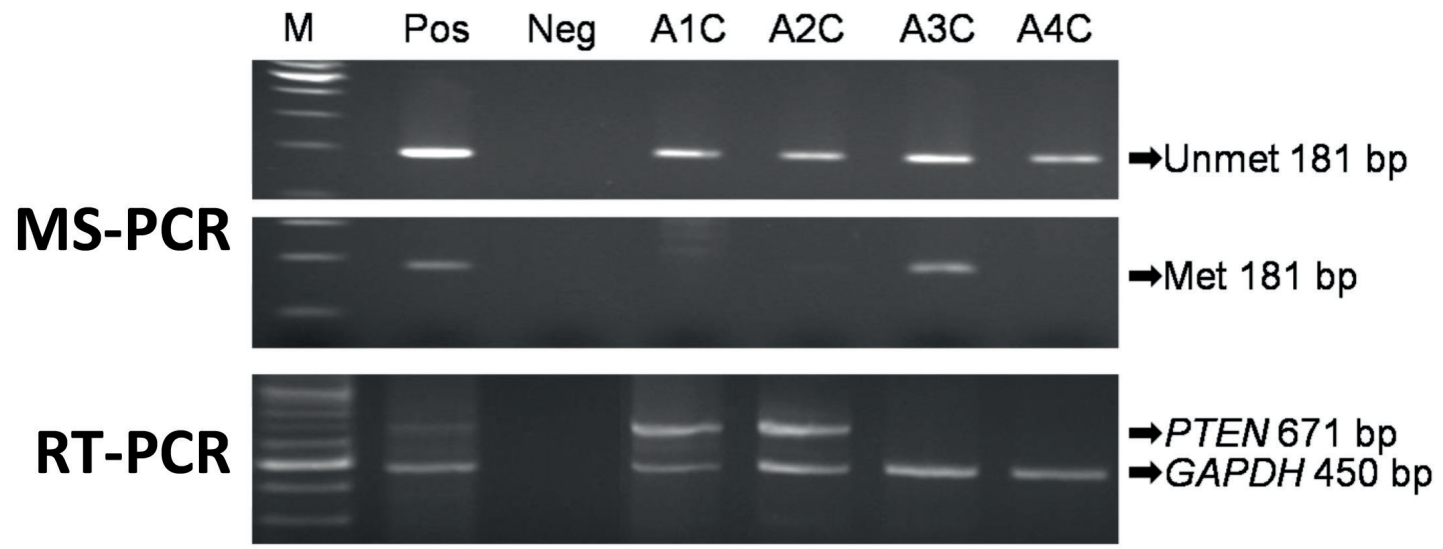

Fig. 3: MS-PCR and RT-PCR analyses of promoter methylation and mRNA expression of PTEN in primary ameloblastoma cell cultures. The upper and middle panels show MS-PCR results, whereas the bottom panel shows RT-PCR results. Arrows indicate the locations of the expected amplicons. GAPDH was used in RT-PCR as an internal control.

Standard 100 bp marker, M; positive control, Pos: HeLa cell line; negative control, Neg: distilled water; methylation-specific PCR, MS-PCR; reverse transcription polymerase chain reaction, RT-PCR; phosphatase and tensin homolog, PTEN; glyceraldehyde3-phosphate dehydrogenase, GAPDH; unmethylated PCR products, Unmet; methylated PCR products, Met.

\section{Discussion}

Ameloblastoma is considered the most common benign neoplasm of the jaw (1). To prevent local recurrence, patients with ameloblastoma are mostly treated with radical surgery. Understanding the molecular mechanisms that underlie the formation of this tumor may help in developing an alternative and novel treatment for its cure with minimal tissue or bone removal.

$P T E N$, a putative tumor suppressor gene, is commonly mutated in many types of human neoplasms (3). The protein product of PTEN, a lipid phosphatase, negatively regulates the Akt signaling pathway, thereby stimulating cell cycle arrest and apoptosis (13). Kumamoto and Ooya first reported that the PTEN level is significantly lower in ameloblastic tumors than in tooth germs (14). The absence of PTEN in 33.3\% of ameloblastoma samples was subsequently reported (10). These results suggest that the inactivation of PTEN may be involved in the molecular pathogenesis of ameloblastoma. In the present study, we investigated the possible role of PTEN promoter methylation and the associated loss of PTEN expression in a subset of ameloblastoma samples.

To the best of our knowledge, there have been no studies on PTEN promoter in ameloblastoma. Careful analysis of the PTEN promoter has been recommended because it shares a strong homology with the PTEN pseudogene (5). The genomic sequence of the highly conserved and processed PTEN pseudogene (GenBank accession number: AF040103, PTEN pseudogene; AF029308, Homo sapiens chromosome 9 duplication of the T-cell receptor $\beta$ locus and trypsinogen gene families) is $98 \%$ identi- cal to that of PTEN, and this identical sequence is composed of an 841-bp region in the promoter region (15). In the present study, PTEN promoter methylation was performed using methylation-specific primers that do not amplify the highly homologous PTEN pseudogene because these primers lie outside the sequence homology of the PTEN pseudogene.

Promoter methylation is reportedly one of the epigenetic mechanisms underlying the aberrant expression of tumor suppressor genes and contributing to the development of various types of cancers. For example, the methylation of adenomatous polyposis coli promoter is reportedly associated with tumor in the colon and breasts (16). PTEN promoter methylation is also observed in various types of cancers, including gastric, breast, colorectal, and lung cancer (7,8,17-20).

In the present study, PTEN promoter methylation was found in $65 \%(13 / 20)$ of the ameloblastoma samples. However, immunohistochemical staining of PTEN expression was performed in only 10 samples. Of these samples, $3(60 \%)$ of 5 samples with PTEN promoter methylation were associated with loss of PTEN expression, whereas $4(80 \%)$ of 5 samples without PTEN promoter methylation showed PTEN expression. PTEN promoter methylation and decreased PTEN expression were not significantly correlated, indicating that other genetic or epigenetic mechanisms possibly regulate PTEN expression, for example, genetic alterations, transcriptional silencing, post-transcriptional regulation, and modification (21). Previously, PTEN exhibited high frequency of allelic losses (62\%) in ameloblastic 
tumors (9). Moreover, Narayan et al. reported that 5 $(25 \%)$ of 20 samples of solid/multicystic ameloblastoma exhibited gene alterations in exon 5 of PTEN while no $P T E N$ mutation was observed in normal tooth germs. However, associated protein expression was not examined in those samples (22). Based on the two-hit model (23), it may be possible that PTEN promoter methylation and allelic loss play a role in PTEN inactivation since $P T E N$ is a tumor suppressor gene. It is also possible that PTEN promoter methylation, contributing to a decrease in protein expression, depends on the specific tumor type. Previous studies on lung and ovarian cancers did not see a correlation between PTEN promoter methylation and loss of protein expression $(18,24)$.

Notably, in the present study, 2 (40\%) of 5 samples with PTEN promoter methylation showed PTEN expression. This may be attributed to the partial methylation of PTEN at the promoter region. It has been proposed that translational inactivation involves a series of events requiring a sufficient DNA methylation level. The silencing process is then maintained by the spread of methylation (25). This is also supported by the presence of unmethylated bands in several samples following MS-PCR. However, these unmethylated bands also represent normal fibrous tissue stroma in the ameloblastoma samples.

Regarding the in vitro experiment, only 1 (25\%) of 4 primary ameloblastoma cell cultures showed promoter methylation and loss of PTEN transcription. This result is consistent with a previous study on breast cancer; none of the breast cancer cell lines exhibited PTEN promoter methylation (17). Lastly, the limitation of the present study is the small sample size that may not represent ameloblastoma cases and cell lines in general; thus, further studies with a larger sample size are required to confirm our findings.

In conclusion, PTEN promoter methylation was detected in a subset (58.3\%) of ameloblastoma samples; however, it did not significantly contribute to decreased PTEN expression. Other genetic mechanisms possibly underlie the loss of PTEN expression in ameloblastomas.

\section{References}

1. Petrovic ID, Migliacci J, Ganly I, Patel S, Xu B, Ghossein R, et al. Ameloblastomas of the mandible and maxilla. Ear Nose Throat J. 2018;97:E26-e32.

2. Kar IB, Subramanyam RV, Mishra N, Singh AK. Ameloblastic carcinoma: A clinicopathologic dilemma - Report of two cases with total review of literature from 1984 to 2012. Ann Maxillofac Surg. 2014:4:70-7.

3. Li J, Yen C, Liaw D, Podsypanina K, Bose S, Wang SI, et al. PTEN, a putative protein tyrosine phosphatase gene mutated in human brain, breast, and prostate cancer. Science. 1997;275:1943-7.

4. Cully M, You H, Levine AJ, Mak TW. Beyond PTEN mutations: the PI3K pathway as an integrator of multiple inputs during tumorigenesis. Nat Rev Cancer. 2006;6:184-92.
5. Zysman MA, Chapman WB, Bapat B. Considerations when analyzing the methylation status of PTEN tumor suppressor gene. Am J Pathol. 2002;160:795-800.

6. Baeza N, Weller M, Yonekawa Y, Kleihues P, Ohgaki H. PTEN methylation and expression in glioblastomas. Acta Neuropathol. 2003; 106:479-85.

7. Lu YM, Cheng F, Teng LS. The association between phosphatase and tensin homolog hypermethylation and patients with breast cancer, a meta-analysis and literature review. Sci Rep. 2016;6:32723.

8. Akca H, Demiray A, Aslan M, Acikbas I, Tokgun O. Tumour suppressor PTEN enhanced enzyme activity of GPx, SOD and catalase by suppression of PI3K/AKT pathway in non-small cell lung cancer cell lines. J Enzyme Inhib Med Chem. 2013;28:539-44.

9. Nodit L, Barnes L, Childers E, Finkelstein S, Swalsky P, Hunt J. Allelic loss of tumor suppressor genes in ameloblastic tumors. Mod Pathol. 2004;17:1062-7.

10. Scheper MA, Chaisuparat R, Nikitakis NG, Sauk JJ. Expression and alterations of the PTEN / AKT / mTOR pathway in ameloblastomas. Oral Dis. 2008;14:561-8.

11. Soria JC, Lee HY, Lee JI, Wang L, Issa JP, Kemp BL, et al. Lack of PTEN expression in non-small cell lung cancer could be related to promoter methylation. Clin Cancer Res. 2002;8:1178-84.

12. Zhu Z, Jia J, Lu R, Lu Y, Fu Z, Zhao L, et al. Expression of PTEN, p27, p21 and AKT mRNA and protein in human BEL-7402 hepatocarcinoma cells in transplanted tumors of nude mice treated with the tripeptide tyroservatide (YSV). Int J Cancer. 2006;118:1539-44.

13. Song MS, Salmena L, Pandolfi PP. The functions and regulation of the PTEN tumour suppressor. Nat Rev Mol Cell Biol. 2012;13:283-96. 14. Kumamoto H, Ooya K. Immunohistochemical detection of phosphorylated Akt, PI3K, and PTEN in ameloblastic tumors. Oral Dis. 2007:13:461-7.

15. Dahia PL, FitzGerald MG, Zhang X, Marsh DJ, Zheng Z, Pietsch $\mathrm{T}$, et al. A highly conserved processed PTEN pseudogene is located on chromosome band 9p21. Oncogene. 1998;16:2403-6.

16. Bouras E, Karakioulaki M, Bougioukas KI, Aivaliotis M, Tzimagiorgis G, Chourdakis M. Gene promoter methylation and cancer: An umbrella review. Gene. 2019;710:333-40.

17. Khan S, Kumagai T, Vora J, Bose N, Sehgal I, Koeffler PH, et al. PTEN promoter is methylated in a proportion of invasive breast cancers. Int J Cancer. 2004;112:407-10.

18. Marsit CJ, Zheng S, Aldape K, Hinds PW, Nelson HH, Wiencke $\mathrm{JK}$, et al. PTEN expression in non-small-cell lung cancer: evaluating its relation to tumor characteristics, allelic loss, and epigenetic alteration. Hum Pathol. 2005;36:768-76.

19. Xu WT, Yang Z, Lu NH. Roles of PTEN (Phosphatase and Tensin Homolog) in gastric cancer development and progression. Asian Pac J Cancer Prev. 2014;15:17-24.

20. Puerta-Garcia E, Canadas-Garre M, Calleja-Hernandez MA. Molecular biomarkers in colorectal carcinoma. Pharmacogenomics. 2015;16:1189-222

21. Alvarez-Garcia V, Tawil Y, Wise HM, Leslie NR. Mechanisms of PTEN loss in cancer: It's all about diversity. Semin Cancer Biol. 2019;59:66-79.

22. Narayan B, Urs AB, Augustine J, Singh H, Polipalli SK, Kumar $\mathrm{S}$, et al. Genetic alteration of Exon 5 of the PTEN gene in Indian patients with ameloblastoma. Oral Surg Oral Med Oral Pathol Oral Radiol. 2019;127:225-30.

23. Huhns M, Salem T, Schneider B, Krohn M, Linnebacher M, Prall F. PTEN mutation, loss of heterozygosity, promoter methylation and expression in colorectal carcinoma: two hits on the gene? Oncol Rep. 2014;31:2236-44.

24. Martins FC, Santiago I, Trinh A, Xian J, Guo A, Sayal K, et al. Combined image and genomic analysis of high-grade serous ovarian cancer reveals PTEN loss as a common driver event and prognostic classifier. Genome Biol. 2014;15:526.

25. Turker MS. Gene silencing in mammalian cells and the spread of DNA methylation. Oncogene. 2002;21:5388-93. 


\section{Acknowledgements}

We thank Mr. Dusit Bumalee for his assistance in histopathological laboratory.

\section{Funding}

This research was supported by National Science and Technology Development Agency (Grant number FDA-CO-2561-8477-TH).

\section{Conflict of interest}

All authors declare that they have no conflict of interest.

\section{Ethics}

This study was conducted in accordance with the approved human subject research guidelines and was approved by the Institutional Review Board of Faculty of Dentistry/Faculty of Pharmacy, Mahidol University, Thailand (COA. No. MU-DT/PY-IRB 2018/005.1101 and 2018/049.0409). Informed consent was obtained from all patients before the collection of specimens. Data were analyzed anonymously. 